\title{
Evaluation of Cardiac Troponin I as a Predictor of Outcome of Sepsis in Critically Ill Patients
}

\author{
MOHAMED S.M. ZAKI, M.D.; ASHRAF E.A. ELAGAMY, M.D.; WAEL A.M. ABDEL WAHAB, M.D. and \\ MAHMOUD M.M. SENNA, M.Sc.
}

The Department of Anaesthesia, Intensive Care and Pain Management, Faculty of Medicine, Ain Shams University

\begin{abstract}
Background: Sepsis and septic shock are the most common causes of morbidity and mortality in Intensive Care Units in the United States. The cardiovascular abnormalities associated with septic shock, in large part, account for the life-threatening nature of the syndrome. Cardiac troponin I (cTnI) has been shown to be an indicator of myocardial injury and is an accepted prognostic factor of Myocardial Infarction (MI).

Aim of Study: To study the prognostic value of cTnI on mortality and adverse complications in patients with sepsis and septic shock. And to study the relation of cTnI with ICU scoring system (SOFA).

Patients and Methods: This is a comparative cross sectional study, was conducted at Intensive Care Unit (ICU) at Ain Shams University Hospitals and El-Matria Teaching Hospital, on 70 patients with suspected infection who were aged between 18 and 60 years old, over the period of six months from November 2019 to April 2020.

Results: Finally, as regard relation between outcome and 1st sample cTnI in each group; the study on the hand revealed that there was no statistical significant difference between outcome and 1st sample cTnI in Group with positive cTnI, Group II with non-elevated cardiac troponin I.

Conclusion: Based on our results we recommend for further studies in larger patients and longer period of followup to emphasize our conclusion. Sepsis patients with high cTnI levels are usually more critically ill while had the same chance to adverse outcome and less mortality and cTnI level is not a predicator of mortality, further studies in larger patient populations must establish whether elevated troponin may be used as an independent mortality risk factor for intensive care patients without ACS.
\end{abstract}

Key Words: Intensive Care Unit-Myocardial infarctionCardiac troponin I.

Correspondence to: Dr. Mahmoud M.M.

Senna,E-Mail: medo.senna@gmail.com

\section{Introduction}

SEPSIS is a life-threatening condition that arises when the body's response to infection causes injury to its own tissues and organs [1].

"Septicemia", also spelled "septicaemia", and " blood poisoning" referred to the microorganisms or their toxins in the blood and are no longer commonly used. The modern term for this is bacteremia [2].

Systemic Inflammatory Response Syndrome ( SIRS) (criteria had been used to define sepsis. If the SIRS criteria are negative, it is very unlikely the person has sepsis; if it is positive, there is just a moderate probability that the person has sepsis. According to SIRS, there were different levels of sepsis: Sepsis, severe sepsis, and septic shock [3].

SIRS is the presence of two or more of the following: Abnormal body temperature, heart rate, respiratory rate, or blood gas, and white blood cell count.

Sepsis is defined as SIRS in response to an infectious process [4].

In 2016 a new consensus was reached to replace screening by Systemic Inflammatory Response Syndrome (SIRS) with the sequential organ failure assessment score (SOFA score). The sequential organ failure assessment score (SOFA score), previously known as the sepsis-related organ failure assessment score, is used to track a person's status during the stay in an Intensive Care Unit (ICU) to determine the extent of a person's organ function or rate of failure. The score is based on six different conrac ono aach for tho rocniratory sardinvacmilar 
hepatic, coagulation, renal and neurological systems [1].

Sepsis is the leading cause of death in the noncoronary Intensive Care Unit (ICU) and the 10th leading cause of death overall [5].

Cardiac troponin I (cTnI) has been shown to be an indicator of myocardial injury and is an accepted prognostic factor of Myocardial Infarction ( MI) [6].

Although cTnI is cardiac-specific, its release seems not to be limited to cardiac-related events, but is also detectable in other critical clinical conditions, such as trauma, pulmonary embolism, and septic shock [7].

Troponin release in this population occurs in the absence of flow-limiting coronary artery disease, suggesting the presence of mechanisms other than thrombotic coronary artery occlusion, probably a transient loss in membrane integrity with subsequent troponin release or microvascular thromboticinjury [8].

\section{Aim of the work:}

To study the prognostic value of cTnI on mortality and adverse complications in patients with sepsis and septic shock. And to study the relation of cTnI with ICU scoring system (SOFA).

\section{Patients and Methods}

\section{Methods:}

\section{Technical design:}

Study type and setting: This study was comparative cross sectional study between sepsis and septic shock patients with elevated cTnI and others with non elevated cTnI by measurement of cTnI in the first $24 \mathrm{~h}$ from admission to ICU (every 6th hours) and the correlation between the trend of measurements (positive or negative) and the patient's outcome (discharge or death).

Study population: Intensive Care Unit (ICU) at Ain Shams University Hospitals and El-Matria Teaching Hospital.

Study period: Six months from November 2019 to April 2020.

\section{Study population:}

Inclusion criteria: Patients with suspected infection who were aged between 18 and 60 years old admitted to ICU can be promptly identified with qSOFA ("HAT"); i.e. 2 or more of: Hypotension: SBP less than or equal to $100 \mathrm{mmHg}$. Altered mental status (any GCS less than 15). Tachypnoea: RR greater than or equal to 22 (breaths per min). Septic shock clinical criteria: Sepsis and (despite adequate volume resuscitation) both of: Persistent hypotension requiring vasopressors to maintain MAP greater than or equal to $65 \mathrm{mmHg}$, and Lactate greater than or equal to $2 \mathrm{mmol} / \mathrm{L}$. And also using APACHE II: It is applied within 24 hours of admission of a patient to an Intensive Care Unit ( ICU): An integer score from 0 to 71 is computed based on several measurements; higher scores correspond to more severe disease and a higher risk of death.

Exclusion criteria: Excluded from the study were patients with any disease that may be associated with an elevation of cardiac troponins as follows: Ischemic heart. Cardiothoracic trauma or surgery. Dilated cardiomyopathy. LV dysfunction. Pulmonary embolism. Chronic renal failure. Severe trauma. Known exposure to burns or toxic chemicals.

\section{Sampling method:}

Sample size: 70 patients.

Ethical considerations: Participants were enrolled for 6 months at Ain Shams University Hospitals and El-Mataria Teaching Hospital. Patient informed written consent and Local Ethical Committee approval have been obtained before patient's allocation.

\section{Study procedures:}

Full history (if possible): Collected data about: Age and sex. Medications.

Clinical examination: Patients with sepsis were identified: Sepsis is defined as life-threatening organ dysfunction caused by a dysregulated host response to infection. The clinical criteria for sepsis include suspected or documented infection and an acute increase of two or more Sequential Organ Failure Assessment (SOFA) points as a proxy for organ dysfunction. Septic shock is defined as a subset of sepsis in which underlying circulatory and cellular/metabolic abnormalities are profound enough to increase mortality substantially. Septic shock is defined by the clinical criteria of sepsis and vasopressor therapy needed to elevate mean arterial pressure $>\_65 \mathrm{~mm} \mathrm{Hg}$ and lactate $>2 \mathrm{mmol} / \mathrm{L}$ ( $18 \mathrm{mg} / \mathrm{dL}$ ) despite adequate fluid resuscitation [9]. 
Conscious level: Attention. Orientation. Speech. Temperature. Heart rate. Blood pressure. Respiratory rate.

\section{Investigations:}

Radiology: Chest X-ray. Electrocardiogram ( ECG). Echocardiograghy.

Laboratory: Complete blood count. $\mathrm{Na}$ and $\mathrm{K}$ serum level. Coagulation profile. Serum lactate. Arterial blood gases. Liver function tests. Kidney function tests. Cardiac troponin I.

Patients included were classified into two groups based on their serum troponin I levels: Low troponin group (troponin $\leq 0.1 \mathrm{~g} / \mathrm{L}$ ) and elevated troponin group (troponin $>0.1 \mathrm{~g} / \mathrm{L}$ ). There is no clear definition as to what constitutes an elevated troponin in patients with sepsis. Different studies have quoted different levels ranging from $0.1 \mathrm{~g} / \mathrm{L}$ to $1 \mathrm{~g} / \mathrm{L}[\mathbf{1 0}]$. In the study, we defined elevated troponin as $>0.1 \mathrm{~g} / \mathrm{L}$ within $12 \mathrm{~h}$ of admission to the ICU. We have used $>0.1 \mathrm{~g} / \mathrm{L}$ as this was the most commonly used cut-off in the published studies [11]. Troponins were assayed using ADVIA Centaur CP system (Bayer Health Care Diagnostics, New York, USA) using the principles of chemiluminescence. The normal reference range in our laboratory is $0.00-0.05 \mathrm{~g} / \mathrm{L}$.

Administrative and ethical design: A written informed consent was obtained from the parents of all patients of the study. After being informed about the aims and process of the study as well as applicable objectives. The study had been approved by the Local Ethics Committee on research involving human subjects of Faculty of Medicine, Ain Shams University. The study procedures were free from any harmful effects on the participants as well as the service provided. There was no extra fee to be paid by the participants and the investigators covered all the costs in this regard. There had been adequate provisions to maintain privacy of participants and confidentiality as follow: We had been put code number to every participant symbol ling to the name and address that had been kept in a special file. The names of the participants in the research will be hidden.

\section{Data management and statistical analysis:}

Collected data were recorded then presented and analyzed statistically by computer using SPSS version 22 (SPSS Inc. Chicago, IL, U.S.A) as follow: Editing and coding. Data entry in computer. Data were summarized and presented in tables and graphs and summarized as median and mean \pm standard deviation for quantitative variables and as number and percentage for qualitative variables. Data were handled using appropriate statistical tests of significance such as: Shapiro-Wilk's Wtest was applied for checking the normality assumption of continuous variables. Chi-square test and Fisher's exact test, wherever appropriate, were used for data analysis. Independent twosample $t$-tests or Mann-Whitney-U-tests were applied to compare the continuous variables between the two groups. Correlation between: The prognostic value and mortality rate between elevated $\mathrm{cTnI}$ in patients with sepsis and septic shock and others with non-elevated cTnI. Other parameters were assessed with Spearman's correlation test. $p$-value less than 0.05 was considered statistically significant. $p$ - value more than 0.05 was considered not significant.

\section{Results}

Table (1): Comparison between the two studied groups according to demographic data.

\begin{tabular}{|c|c|c|c|c|c|c|}
\hline & \multicolumn{2}{|c|}{$\begin{array}{c}\text { Group I } \\
(n=35)\end{array}$} & \multicolumn{2}{|c|}{$\begin{array}{c}\text { Group II } \\
(\mathrm{n}=35)\end{array}$} & \multirow{2}{*}{$\begin{array}{c}\text { Test } \\
\text { of sig. }\end{array}$} & \multirow{2}{*}{$p$} \\
\hline & No. & $\%$ & No. & $\%$ & & \\
\hline \multicolumn{7}{|l|}{ Gender: } \\
\hline Male & 22 & 62.9 & 19 & 54.3 & $\chi^{2}=$ & 0.467 \\
\hline Female & 13 & 37.1 & 16 & 45.7 & 0.530 & \\
\hline \multicolumn{7}{|l|}{ Age (years): } \\
\hline Min.-max. & \multicolumn{2}{|c|}{$18.0-60.0$} & \multicolumn{2}{|c|}{$18.0-70.0$} & $t=$ & 0.230 \\
\hline Mean \pm SD. & \multicolumn{2}{|c|}{$45.37 \pm 13.10$} & \multicolumn{2}{|c|}{$41.17 \pm 15.79$} & 1.211 & \\
\hline \multirow[t]{2}{*}{ Median (IQR) } & \multicolumn{2}{|c|}{50.0} & \multicolumn{2}{|c|}{45.0} & & \\
\hline & \multicolumn{2}{|c|}{$(38.50-55.50)$} & \multicolumn{2}{|c|}{$(25.0-55.50)$} & & \\
\hline
\end{tabular}

Table (2): Comparison between the two studied groups according to medications.

\begin{tabular}{|c|c|c|c|c|c|c|}
\hline & \multicolumn{2}{|c|}{$\begin{array}{l}\text { Group I } \\
(\mathrm{n}=35)\end{array}$} & \multicolumn{2}{|c|}{$\begin{array}{c}\text { Group II } \\
(\mathrm{n}=35)\end{array}$} & \multirow[t]{2}{*}{$\chi^{2}$} & \multirow[t]{2}{*}{$p$} \\
\hline & No. & $\%$ & No. & $\%$ & & \\
\hline \multicolumn{7}{|c|}{ Medications: } \\
\hline No & 11 & 31.4 & 15 & 42.9 & 0.979 & 0.322 \\
\hline Yes & 24 & 68.6 & 20 & 57.1 & & \\
\hline $\mathrm{BB}$ & 11 & 45.8 & 7 & 35.0 & 0.530 & 0.467 \\
\hline Metformine & 4 & 16.7 & 2 & 10.0 & 0.412 & $\mathrm{FE} p=0.673$ \\
\hline Statin & 5 & 20.8 & 1 & 5.0 & 2.322 & $\mathrm{FE} p=0.198$ \\
\hline $\mathrm{ACEi}$ & 7 & 29.2 & 5 & 25.0 & 0.095 & 0.757 \\
\hline Insulin & 7 & 29.2 & 7 & 35.0 & 0.171 & 0.679 \\
\hline CCB & 4 & 16.7 & 4 & 20.0 & 0.081 & $\mathrm{FE} p=1.000$ \\
\hline Diuretic & 1 & 4.2 & 3 & 15.0 & 1.549 & $\mathrm{FE} p=0.316$ \\
\hline ARB & 2 & 8.3 & 3 & 15.0 & 0.481 & $\mathrm{FE} p=0.646$ \\
\hline
\end{tabular}

$\chi^{2}$ : Chi square test.

FE : Fisher Exact.

$p: p$-value for comparing between the studied groups. 
Table (3): Comparison between the two studied groups according to risk factors.

\begin{tabular}{|c|c|c|c|c|c|c|}
\hline \multirow{2}{*}{ Risk factors } & \multicolumn{2}{|c|}{$\begin{array}{l}\text { Group I } \\
(\mathrm{n}=35)\end{array}$} & \multicolumn{2}{|c|}{$\begin{array}{c}\text { Group II } \\
(\mathrm{n}=35)\end{array}$} & \multirow{2}{*}{$\chi$} & \multirow{2}{*}{$p$} \\
\hline & No. & $\%$ & No. & $\%$ & & \\
\hline \multicolumn{7}{|l|}{$H T N:$} \\
\hline Negative & 9 & 25.7 & 14 & 40.0 & 1.619 & 0.203 \\
\hline Positive & 26 & 74.3 & 21 & 60.0 & & \\
\hline \multicolumn{7}{|c|}{ Dyslipidemia: } \\
\hline Negative & 15 & 42.9 & 15 & 42.9 & 0.000 & 1.000 \\
\hline Positive & 20 & 57.1 & 20 & 57.1 & & \\
\hline \multicolumn{7}{|l|}{$D M:$} \\
\hline Negative & 21 & 60.0 & 23 & 65.7 & 0.245 & 0.621 \\
\hline Positive & 14 & 40.0 & 12 & 34.3 & & \\
\hline
\end{tabular}

$\chi^{2}$ : Chi square test.

$p: p$-value for comparing between the studied groups.

Table (4): Comparison between the two studied groups according to vital signs.

\begin{tabular}{|c|c|c|c|}
\hline Vital signs & Group I & Group II & $p$ \\
\hline $\begin{array}{l}\text { HR: } \\
\text { Min.-max. } \\
\text { Mean } \pm \text { SD. } \\
\text { Median (IQR) }\end{array}$ & $\begin{array}{l}(\mathrm{n}=35) \\
92.0-114.0 \\
103.0 \pm 5.01 \\
102.0 \\
(100.0-106.0)\end{array}$ & $\begin{array}{l}(n=35) \\
96.0-125.0 \\
110.6 \pm 8.10 \\
110.0 \\
(105.5-117.5)\end{array}$ & $4.722 *<0.001 *$ \\
\hline $\begin{array}{l}\text { Systolic blood } \\
\text { pressure:Min.- } \\
\text { max. Mean } \pm \\
\text { SD. Median ( } \\
\text { IQR) }\end{array}$ & $\begin{array}{l}(\mathrm{n}=35) \\
70.0-100.0 \\
88.14 \pm 9.93 \\
90.0 \\
(80.0-100.0)\end{array}$ & $\begin{array}{l}(\mathrm{n}=28) \\
60.0-80.0 \\
67.86 \pm 8.44 \\
65.0 \\
(60.0-75.0)\end{array}$ & $8.603 *<0.001 *$ \\
\hline $\begin{array}{l}\text { Diastolic blood } \\
\text { pressure:Min.- } \\
\text { max. Mean } \pm \\
\text { SD. Median ( } \\
\text { IQR) }\end{array}$ & $\begin{array}{l}30.0-70.0 \\
50.86 \pm 10.11 \\
50.0 \\
(45.0-60.0)\end{array}$ & $\begin{array}{l}(\mathrm{n}=26) \\
4.0-50.0 \\
33.62 \pm 11.20 \\
30.0 \\
(30.0-40.0)\end{array}$ & $6.291 *<0.001 *$ \\
\hline $\begin{array}{l}\text { Respiratory rate: } \\
\text { Min.-max. } \\
\text { Mean } \pm \text { SD. } \\
\text { Median (IQR) }\end{array}$ & $\begin{array}{l}(\mathrm{n}=35) \\
22.0-31.0 \\
25.69 \pm 2.59 \\
25.0 \\
(24.0-27.50)\end{array}$ & $\begin{array}{l}(\mathrm{n}=35) \\
22.0-35.0 \\
28.20 \pm 3.60 \\
29.0 \\
(25.0-30.0)\end{array}$ & $3.353 * 0.001 *$ \\
\hline $\begin{array}{l}\text { Temp.: } \\
\text { Min.-max. } \\
\text { Mean } \pm \text { SD. } \\
\text { Median (IQR) }\end{array}$ & $\begin{array}{l}(\mathrm{n}=35) \\
38.0-40.0 \\
38.83 \pm 0.62 \\
39.0 \\
(38.50-39.0)\end{array}$ & $\begin{array}{l}(\mathrm{n}=35) \\
38.0-40.0 \\
38.96 \pm 0.70 \\
39.0 \\
(38.50-39.50)\end{array}$ & 0.8140 .418 \\
\hline
\end{tabular}

$t:$ Student $t$-test

$p$ : $p$-value for comparing between the studied groups.

*: Statistically significant at $p \leq 0.05$.

Table (5): Comparison between the two studied groups according to GCS.

\begin{tabular}{lcccc}
\hline GCS & $\begin{array}{c}\text { Group 1 } \\
(\mathrm{n}=35)\end{array}$ & $\begin{array}{c}\text { Group II } \\
(\mathrm{n}=35)\end{array}$ & $t$ & $p$ \\
\hline Min.-max. & $3.0-14.0$ & $3.0-14.0$ & 1.1870 .239 \\
Mean \pm SD. & $9.63 \pm 2.71$ & $10.40 \pm 2.72$ & \\
Median (IQR) & $10.0(8.0-12.0)$ & $10.0(9.0-12.0)$ & &
\end{tabular}

$t$ : Student $t$-test.

$p$ : $p$-value for comparing between the studied groups.
Table (6): Comparison between the two studied groups according to radiological investigations.

\begin{tabular}{|c|c|c|c|c|c|c|}
\hline \multirow[t]{2}{*}{ Radiological Ix. } & \multicolumn{2}{|c|}{$\begin{array}{l}\text { Group I } \\
(n=35)\end{array}$} & \multicolumn{2}{|c|}{$\begin{array}{c}\text { Group II } \\
(\mathrm{n}=35)\end{array}$} & \multirow[t]{2}{*}{$\begin{array}{c}\text { Test of } \\
\text { sig. }\end{array}$} & \multirow{2}{*}{$p$} \\
\hline & No. & $\%$ & No. & $\%$ & & \\
\hline \multicolumn{7}{|l|}{$C X R:$} \\
\hline NAD & 27 & 77.1 & 17 & 48.6 & \multirow{2}{*}{$\begin{array}{l}\chi^{2}= \\
6.119 *\end{array}$} & \multirow[t]{2}{*}{0.013} \\
\hline Chest infection & 8 & 22.9 & 18 & 51.4 & & \\
\hline \multicolumn{7}{|l|}{$E C G:$} \\
\hline NAD & 7 & 20.0 & 6 & 17.1 & \multirow{2}{*}{$\begin{array}{l}\chi^{2}= \\
0.094\end{array}$} & \multirow[t]{2}{*}{0.759} \\
\hline ST & 28 & 80.0 & 29 & 82.9 & & \\
\hline \multicolumn{7}{|l|}{ Echo.: } \\
\hline \multicolumn{7}{|l|}{$E F$} \\
\hline Min.-max. & \multirow{3}{*}{\multicolumn{2}{|c|}{$\begin{array}{l}50.0-75.0 \\
65.06 \pm 6.65 \\
65.0 \\
(60.0-70.50)\end{array}$}} & \multirow{3}{*}{\multicolumn{2}{|c|}{$\begin{array}{l}55.0-75.0 \\
63.0 \pm 5 \\
9760.0 \\
(60.0-67.50)\end{array}$}} & \multirow{3}{*}{$\begin{array}{l}t= \\
1.362\end{array}$} & \multirow[t]{3}{*}{0.178} \\
\hline Mean \pm SD. & & & & & & \\
\hline Median (IQR) & & & & & & \\
\hline \multirow{2}{*}{\multicolumn{7}{|c|}{ Valves: }} \\
\hline $\begin{array}{l}\text { Valves: } \\
\text { NAD }\end{array}$ & & & & & & \multirow{5}{*}{$\begin{array}{l}\mathrm{MC}_{p}= \\
0.754\end{array}$} \\
\hline Mild AS & 3 & $\begin{array}{l}02.9 \\
8.6\end{array}$ & 1 & $\begin{array}{l}0.9 \\
2.9\end{array}$ & \multirow{4}{*}{$\begin{array}{l}\chi^{2}= \\
3.087\end{array}$} & \\
\hline Mild AR & 0 & 0.0 & 1 & 2.9 & & \\
\hline Mild MR & 2 & 5.7 & 3 & 8.6 & & \\
\hline Mod MR & 1 & 2.9 & & 0.0 & & \\
\hline
\end{tabular}

$R W M A$

irin

$\chi^{2}$ : Chi square test.

$\begin{array}{lllll}35 & 100.0 & 35 & 100.0 & -\end{array}$

$t$ : Student $t$-test. $\quad *$ : Statistically significant at $p \leq 0.05$

$p: p$-value for comparing between the studied groups.

Table (7): Comparison between the two studied groups according to $\mathrm{CBC}$

\begin{tabular}{|c|c|c|c|c|}
\hline СВС & $\begin{array}{l}\text { Group I } \\
(\mathrm{n}=35)\end{array}$ & $\begin{array}{l}\text { Group II } \\
(\mathrm{n}=35)\end{array}$ & $\begin{array}{l}\text { Test of } \\
\text { sig. }\end{array}$ & $p$ \\
\hline \multicolumn{5}{|l|}{$H B:$} \\
\hline $\begin{array}{l}\text { Min.-max. } \\
\text { Mean } \pm \text { SD. } \\
\text { Median (IQR) }\end{array}$ & $\begin{array}{l}8.50-12.0 \\
10.31 \pm 1.17 \\
10.0 \\
(9.25-11.50)\end{array}$ & $\begin{array}{l}8.0-12.0 \\
9.94 \pm 1.04 \\
10.00 \\
(9.25-10.50)\end{array}$ & $\begin{array}{l}t= \\
1.403\end{array}$ & 0.165 \\
\hline \multicolumn{5}{|l|}{ WBCs $\left(\begin{array}{ll}X & 10^{3}\end{array}\right):$} \\
\hline $\begin{array}{l}\text { Min.-max. } \\
\text { Mean } \pm \text { SD. } \\
\text { Median (IQR) }\end{array}$ & $\begin{array}{l}120.0-250.0 \\
152.7 \pm 30 . \\
33150.0 \\
(130.0-160.0)\end{array}$ & $\begin{array}{l}130.0-270.0 \\
184.3 \pm 41.07 \\
170.0 \\
(155.0-210.0)\end{array}$ & $\begin{array}{l}t= \\
3.65 \gamma^{*}\end{array}$ & $0.001^{*}$ \\
\hline \multicolumn{5}{|l|}{$P L T:$} \\
\hline $\begin{array}{l}\text { Min.-max. } \\
\text { Mean } \pm \text { SD. } \\
\text { Median (IQR) }\end{array}$ & $\begin{array}{l}150.0-450.0 \\
229.4 \pm 76.83 \\
200.0 \\
(180.0-255.0)\end{array}$ & $\begin{array}{l}150.0-310.0 \\
207.1 \pm 41.80 \\
195.0 \\
(180.0-235.0)\end{array}$ & $\begin{array}{l}U= \\
J U . J U\end{array}$ & 0.400 \\
\hline
\end{tabular}

$t:$ Student $t$-test. U: Mann Whitney test.

$p: p$-value for comparing between the studied groups.

: Statistically significant at $p \leq 0.05$.

Table (8): Comparison between the two studied groups according to electrolytes.

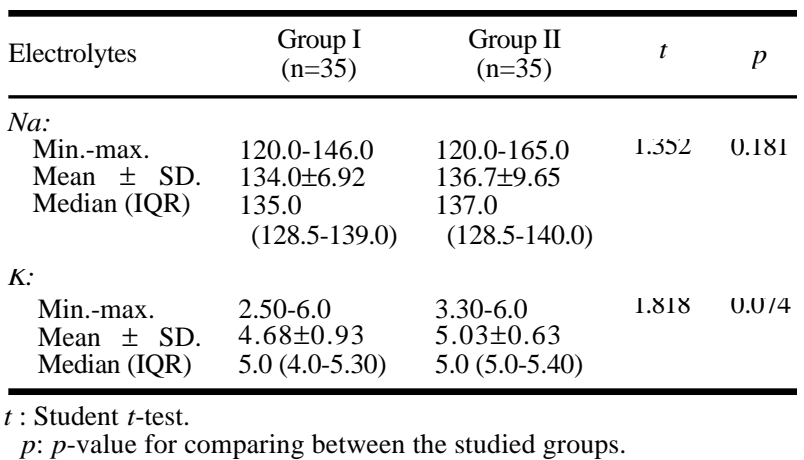


Table (9): Comparison between the two studied groups according to INR.

\begin{tabular}{|c|c|c|c|}
\hline INR & Group I $(n=35$ & Group II $(n=35)$ & $p$ \\
\hline Min.-max. & $1.0-1.40$ & $1.0-1.50$ & 0.4490 .665 \\
\hline Mean \pm SD & $1.10 \pm 0.12$ & $1.11 \pm 0.15$ & \\
\hline Median (IQR) & $1.10(1.0-1.20)$ & $1.0(1.0-1.20)$ & \\
\hline
\end{tabular}

Table (10): Comparison between the two studied groups according to liver function.

\begin{tabular}{|c|c|c|c|}
\hline \multicolumn{3}{|c|}{ Liver function Group I $(n=35)$ Group II $(n=35)$} & $\mathrm{U}$ \\
\hline \multicolumn{4}{|l|}{$\overline{S G P T:}$} \\
\hline $\begin{array}{l}\text { Min.-max. } \\
\text { Mean } \pm \text { SD. } \\
\text { Median (IQR) }\end{array}$ & $\begin{array}{l}15.0-300.0 \\
93.57 \pm 77.87 \\
75.0 \\
(34.0-119.5)\end{array}$ & $\begin{array}{l}13.0-240.0 \\
91.69 \pm 60.86 \\
75.0 \\
(60.0-115.0)\end{array}$ & 580.5000 .707 \\
\hline \multicolumn{4}{|l|}{ SGOT: } \\
\hline $\begin{array}{l}\text { Min.-max. } \\
\text { Mean } \pm \text { SD. } \\
\text { Median (IQR) }\end{array}$ & $\begin{array}{l}8.0-320.0 \\
90.51 \pm 76.93 \\
73.0 \\
(33.0-122.5)\end{array}$ & $\begin{array}{l}12.0-236.0 \\
71.31 \pm 54.66 \\
62.0 \\
(28.50-96.50)\end{array}$ & 539.5000 .391 \\
\hline
\end{tabular}

$\mathrm{U}$ : Mann Whitney test.

$p: p$-value for comparing between the studied groups.

Table (11): Comparison between the two studied groups according to serum creatinine and serum

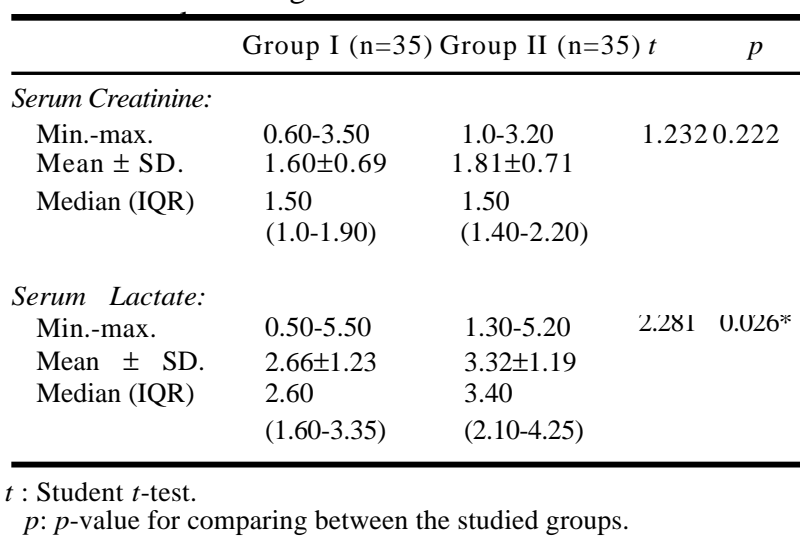

Table (12): Comparison between the two studied groups according to ABG.

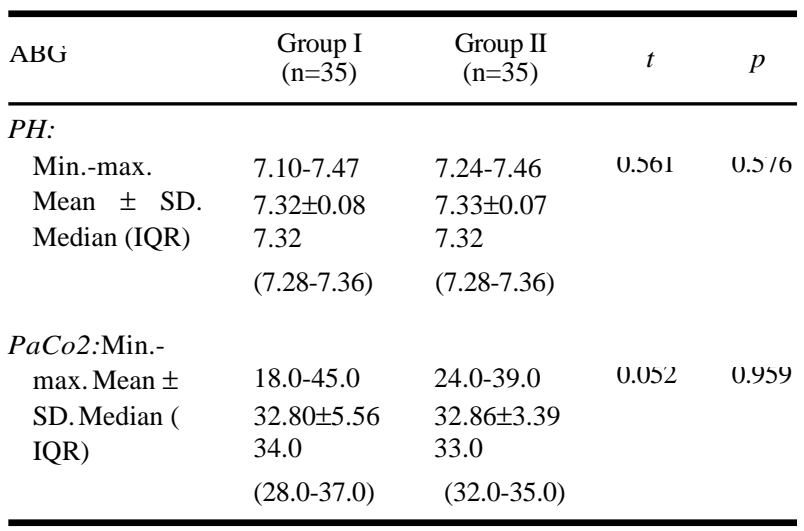

$t:$ Student $t$-test.

$p$ : $p$-value for comparing between the studied groups.

Table (13): Comparison between the two studied groups according to cTnI.

\begin{tabular}{|c|c|c|c|c|}
\hline cInI & Group I $(\mathrm{n}=35)$ & Group II ( $\mathrm{n}=35)$ & $\mathrm{U}$ & $p$ \\
\hline sample: & & & & \\
\hline $\begin{array}{l}\text { Min.-max. } \\
\text { Mean } \pm \text { SD. } \\
\text { Median (IQR) }\end{array}$ & $\begin{array}{c}0.04-0.46 \\
0.20 \pm 0.11 \\
0.19(0.11-0.27)\end{array}$ & $\begin{array}{c}0.11-3.82 \\
2.12 \pm 1.18 \\
2.20(1.08-3.11)\end{array}$ & $54.500^{*}$ & $<0.0011^{*}$ \\
\hline
\end{tabular}

2nd sample:

$\begin{array}{llll}\text { Min.- } & \text { Max. } 0.02-0.49 & 0.08-4.87 & 88.500^{*}<0.001^{*}\end{array}$

Mean \pm SD. $0.21 \pm 0.13 \quad 1.80 \pm 1.29$

Median (IQR) $\quad 0.20(0.10-0.31) \quad 1.58(0.82-2.68)$

3rd sample:

Min.-max. $\quad 0.05-0.61 \quad 0.09-4.83 \quad 12 / .000^{*}<0.001 *$

Mean \pm SD. $0.25 \pm 0.12 \quad 1.79 \pm 1.33$

Median (IQR) $\quad 0.26(0.17-0.34) \quad 1.51(0.43-2.74)$

4th sample:

Min.-max. $\quad 0.02-0.54 \quad 0.17-4.68 \quad 25.000^{*}<0.001 *$

Mean \pm SD. $0.24 \pm 0.12 \quad 2.08 \pm 1.17$

Median (IQR) $\quad 0.21(0.15-0.31) \quad 1.87(1.17-3.06)$

U: Mann Whitney test.

$p: p$-value for comparing between the studied groups

$*$ : Statistically significant at $p \leq 0.05$.

Table (14): Comparison between the different studied samples according to cTnI in each group.

\begin{tabular}{|c|c|c|c|c|c|}
\hline cTnI & 1st sample & 2nd sample & 3rd sample & 4th sample & Fr \\
\hline \multicolumn{6}{|l|}{ Group I $(n=35)$ : } \\
\hline Min.-max. & $0.04-0.460 .20 \pm$ & 0.02-0.49 & $0.05-0.61$ & $0.02-0.54$ & \multirow[t]{3}{*}{2.3760 .498} \\
\hline Mean \pm SD. & $0.110 .19(0.11-$ & $0.21 \pm 0.13$ & $0.25 \pm 0.12$ & $0.24 \pm 0.12$ & \\
\hline Median (IQR) & $0.27)$ & $0.20(0.10-0.31)$ & $0.26(0.17-0.34)$ & $0.21(0.15-0.31)$ & \\
\hline \multicolumn{6}{|l|}{ Group II $(n=35)$ : } \\
\hline Min.-max. & $0.11-3.82$ & $0.08-4.87$ & $0.09-4.83$ & $0.17-4.68$ & \multirow[t]{3}{*}{4.3030 .231} \\
\hline Mean \pm SD & $2.12 \pm 1.18$ & $1.80 \pm 1.29$ & $1.79 \pm 1.33$ & $2.08 \pm 1.17$ & \\
\hline Median (IQR) & $2.20(1.08-3.11)$ & $1.58(0.82-2.68)$ & $1.51(0.43-2.74)$ & $1.87(1.17-3.06)$ & \\
\hline
\end{tabular}

Fr: Friedman test

$p: p$-value for comparing between the different samples in each group. 
Table (15): Comparison between the two studied groups according to SOFA score.

\begin{tabular}{lccc}
\hline SOFA score & Group I $(\mathrm{n}=35)$ Group II $(\mathrm{n}=35)$ & $t$ & $p$ \\
\hline $\begin{array}{l}\text { On admission: } \\
\text { Min.-max. } \\
<0.001 *\end{array}$ & $2.0-11.0$ & $11.0-19.0$ & $13.127^{*}$ \\
Mean \pm SD. $6.83 \pm 2.72$ & $14.83 \pm 2.37$ & \\
Median (IQR) $7.0(5.0-9.0)$ & $15.0(13.0-16.50)$ & \\
$\begin{array}{l}\text { Day 2: } \\
\text { Min.-max. } \\
<0.001 *\end{array}$ & & \\
Mean \pm SD. $6.91 \pm 2.05$ & $15.03 \pm 3.05$ & $13.055^{*}$ \\
p 0 & 0.884 & 0.771 & \\
\hline
\end{tabular}

$t$ : Student $\boldsymbol{t}$-test.

$p: p$-value for comparing between the studied groups.

$p 0: p$-value for paired $t$-test for comparing between on admission and day 2.

* : Statistically significant at $p \leq 0.05$.

Table (16): Comparison between the two studied groups according to APACHE II.

\begin{tabular}{lccc}
\hline APACHE II & Group I $(\mathrm{n}=35)$ Group II $(\mathrm{n}=35)$ & $t$ & $p$ \\
\hline Min.-max. & $12.0-24.0$ & $16.0-50.0$ & $10.020^{*}<0.001 *$ \\
Mean \pm S D. & $17.63 \pm 3.64$ & $34.37 \pm 9.19$ & \\
Median (IQR) & $17.0(15.0-21.0)$ & $34.0(27.50-40.0)$ & \\
\hline$t:$ Student $t$-test. \\
$p: p$-value for comparing between the studied groups.
\end{tabular}

Table (17): Relation between outcome and 1 st sample cTnI in each group.

\begin{tabular}{|c|c|c|c|c|}
\hline \multirow{2}{*}{ 1st sample cTnI } & \multicolumn{2}{|c|}{ Outcome } & \multirow{2}{*}{$\mathrm{U}$} & \multirow{2}{*}{$p$} \\
\hline & Alive & Died & & \\
\hline Group I $(n=35)$ : & $(n=15)$ & $(\mathrm{n}=20)$ & & \\
\hline $\begin{array}{l}\text { Min.-max. } \\
\text { Mean } \pm \text { SD. }\end{array}$ & $\begin{array}{l}0.06-0.35 \\
0.20 \pm 0.10 \\
0.19\end{array}$ & $\begin{array}{l}0.04-0.46 \\
0.20 \pm 0.13 \\
0.19\end{array}$ & 137.0 & 0.681 \\
\hline Group II $(n=35)$ : & $(n=7)$ & $(n=28)$ & & \\
\hline $\begin{array}{l}\text { Min.-max. } \\
\text { Mean } \pm \text { SD. } \\
\text { Median }\end{array}$ & $\begin{array}{l}0.11-3.55 \\
1.73 \pm 1.37 \\
2.01\end{array}$ & $\begin{array}{l}0.13-3.82 \\
2.22 \pm 1.13 \\
2.26\end{array}$ & 73.0 & 0.320 \\
\hline Total $(n=70)$ : & $(n=22)$ & $(n=48)$ & & \\
\hline $\begin{array}{l}\text { Min.-max. } \\
\text { Mean } \pm \text { SD. } \\
\text { Median }\end{array}$ & $\begin{array}{l}0.06-3.55 \\
0.69 \pm 1.04 \\
0.26\end{array}$ & $\begin{array}{l}0.04-3.82 \\
1.38 \pm 1.33 \\
0.84\end{array}$ & 374.50 & 0.052 \\
\hline
\end{tabular}

$\mathrm{U}$ : Mann Whitney test.

$p: p$-value for association between different categories.

\section{Discussion}

Sepsis is the leading cause of death in the noncoronary Intensive Care Unit (ICU) and the 10th leading cause of death overall. Cardiac troponin I ( cTnI) has been shown to be an indicator of myocardial injury and is an accepted prognostic factor of Myocardial Infarction (MI). Although cTnI is cardiac-specific, its release seems not to be limited to cardiac-related events, but is also detectable in other critical clinical conditions, such as trauma, pulmonary embolism, and severe sepsis [5].
The elevation of cardiac troponins in patients with sepsis, and septic shock has been shown to indicate a poor prognosis. Troponin release in this population occurs in the absence of flow-limiting coronary artery disease, suggesting the presence of mechanisms other than thrombotic coronary artery occlusion, probably a transient loss in membrane integrity with subsequent troponin release or micro-vascular thrombotic injury [8].

The aim of the study was to evaluate the prognostic value of cTnI on mortality and adverse complications in patients with sepsis and septic shock, and to study the relation of cTnI with ICU scoring system (SOFA).

This was comparative cross sectional study between sepsis and septic shock patients with elevated cTnI and others with non-elevated cTnI by measurement of cTnI in the first 24th from admission to ICU (every 6th hours) and the correlation between the trend of measurements (positive or negative) and the patient's outcome (discharge or death).

This study was conducted on 35 as Group I and 35 as Group II. Clinical and laboratory data of the 2 groups, Group I included 22 males (62.9\%) and 13 females $(37.1 \%)$. Their age ranged from 18.0 to 60.0 years (mean \pm SD $45.37 \pm 13.10$ ) and Group II included 19 males (54.3\%) and 16 females $(45.7 \%)$. Their age ranged from 18.0 to 70 . 0 years (mean \pm SD 41.17 \pm 15.79 ), meanwhile; we found that there was no statistical significant difference between two groups as regard gender and age.

In agreement with our findings, a prospective comparative study of Salah Eldeen et al., [12] which was conducted on forty five patients admitted to the ICU with sepsis or septic shock. Then patients were divided into 2 groups; group 1: Included 20 patients with positive cTnI (mean age $58 \pm 18.9 \mathrm{yrs}$, $40 \%$ males) and group 2: Included 25 patients with negative cTnI (mean age 52 $\pm 19.3 \mathrm{yrs}, 64 \%$ males), comparisons between 2 groups were done according to all demographic data and revealed that there was no statistical significant difference between them.

Furthermore, as regard medications used, that there was no statistical significant difference between groups as regard medications, also there was no statistical significant differences between groups as regard risk factors as hypertension, DM or dyslipidemia.

In agreement with our findings, the study of Salah Fldeen et al r101 renorted that there were 
no statistical significant differences between groups as regard HTN, DM and vasopressors.

In the present study; we assessed the vital signs of the studied groups and revealed that there was high statistical significant difference between groups as regard HR, systolic blood pressure, diastolic blood pressure and respiratory rate were $p$ $<0.001$. But there was no statistical significant difference between groups as regard Temp.

In comparison with the our findings, the study of Khalaf et al., [13] reported that mean of SBP was $80 \pm 13.3$ in group 1 and was $118.8 \pm 30.5$ in group 2, and there was highly statistical significant difference between groups as regard systolic blood pressure and MAP, while they found that Temp was $38.1 \pm 1.4$ group 1 and was $37.8 \pm 0.7$, and there was no statistical significant difference between groups as regard Temp where $p$-value $=0.49$.

In the present study; mean \pm SD. of GCS in group 1 was $9.63 \pm 2.71$, and was $10.40 \pm 2.72$ in group 2, and there was no statistical significant difference between groups as regard GCS.

Knaus et al., [14] evaluating the definition of sepsis and sepsis syndrome stated that patients with low GCS had a higher mortality rate. Similarly, a prospective case series study of Eidelman et al., [15] conducted on 1996 reported that septic encephalopathy was associated with high risk of mortality proportionally correlated with the GCS, researchers reported patients with a GCS of 15 had $16 \%$ mortality, those with a score of 13 to 14 had $20 \%$ mortality, those with GSC of 9 to 12 had $50 \%$ mortality, and those with GCS of 3 to 8 had $63 \%$ mortality $(p<.05)$.

In the current study, we found that there was no statistical significant difference between groups as regard RWMA, EF and valves but there was high statistical significant difference between groups as regard CXR were $p=0.040$.

On the other hand, as regard laboratory findings, the present study demonstrated that there was no statistical significant difference between groups as regard $\mathrm{HB}$ and PLT but there was high statistical significant difference between groups as regard WBCs $\left(\mathrm{X} \mathrm{10}{ }^{3}\right.$ ) were $p=0.001$, also; we revealed that there was no statistical significant difference between groups as regard $\mathrm{Na}$ and $\mathrm{K}$, and there was no statistical significant difference between groups as regard INR, meanwhile, we found that there was no statistical significant difference between groups as regard SGPT and SGOT, additionally;

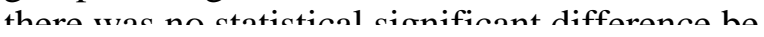

tween groups as regard serum creatinine. But there was high statistical significant difference between groups according to serum lactate.

Troponin I is a cardiac-specific molecule that is released into the systemic circulation following myocardial cell injury. Sepsis and inflammation are the leading non-cardiac causes of elevated troponin levels during critical illness. In sepsis, the heart undergoes physiologic and metabolic changes, including altered coronary blood flow, reduced oxygen extraction, regional and global wall hypokinesia, and ventricular dilatation [16]. Coronary blood flow is often increased. In this situation, a potential explanation for troponin release is cellular ischemia due to microcirculatory changes within the myocardium [17]. Circulating inflammatory cytokines appear to contribute through direct myocytotoxic effects, perturbations in microvascular flow and effects on cell permeability. Regional and global wall hypokinesia resulting in increased cardiac filling pressures and ventricular dilatation may also play a role [16].

In the present study, we found that mean \pm SD. of cTnI in group 1 in ${ }^{1 \text { st }}$ sample was $0.20 \pm 0$. 11 and was $2.12 \pm 1.18$ in group 2 , mean \pm SD. of cTnI in group 1 in 2 nd sample was $0.21 \pm 0.13$, and was $1.80 \pm 1.29$ in group 2 , and there was highly statistical significant difference between groups as regard 1st sample, 2nd sample, 3rd sample and 4th sample of cTnI where $p<0.0016$, also in the present study, we revealed that there was no statistical significant difference between studied samples as regard Group I and Group II.

Previous studies have reported a wide range in prevalence of troponin elevation in critically patients. A meta-analysis by Lim et al., [11] showed that in 20 studies, elevated troponin was found in a median of $43 \%$ (IQR $21 \%$ to $59 \%$ ) of 3,278 patients.

Comparison between groups regarding to SOFA score in the current study revealed that there was high statistical significant difference between groups as regard SOFA score on admission and Day 2 were $p<0.001$.

In agreement with our findings, the study of Gupta et al., [18] reported that the mean SOFA score at admission and 48 hours were 7.09 \pm 3.81 and $7.88 \pm 3.71$ respectively, and there was high statistical significant difference between groups as regard SOFA score on admission and Day 2 were $p<0.001$. 
Another study of Salah Eldeen et al., [12] reported that mean of SOFA on admission was $14.9 \pm 4.2$ in group $\mathrm{I}$, and was $6.96 \pm 4.5$ in group II, and there was highly statistical significant difference between groups where $p=0.0001$, while mean of SOFA at 48 hours in group II was $15.8 \pm 5$. 4 , and was 5.5 \pm 4.4 in group II, and there high statistical significant difference between groups as regard SOFA score on admission and Day 2 were $p$ $<0.001$.

Furthermore, in the current study; mean \pm SD. of APACHE II was $17.63 \pm 3.64$ in group I and was $34.37 \pm 9.19$ in group II, and there was high statistical significant difference between groups as regard PACHE II where $p<0.001$.

Comparing to the study of Salah Eldeen et al., [ 12] which reported that patients with elevated cTnI were more critically ill which appear by higher APACHE II $(34.6 \pm 10.9$ vs. $17.8 \pm 5.4, p$ value $=0.001)$.

These results are similar to Mehta et al., [7] results, where cTnI positive patients showed significantly higher APACHE II score and higher mortality (56\% vs. $24 \%, p=0.04$ ), also Aziz et al., [ 19] demonstrated that Troponin I-positive group had higher APACHE II score ( $30 \pm 6$ vs. $22 \pm 4$. 7) on admission.

In addition to above findings, the preset study revealed that there was high statistical significant difference between groups as regard outcome with higher mortality among group II where $p=0.039$.

Our results regarding mortality are in contrast to the study of Salah Eldeen et al., [12] which showed that also mortality was significantly high in group 1 with positive $\mathrm{C}$ troponin than group 2 ( $90 \%$ vs. $60 \%, p$-value $=0.024)$.

The previous results are in comparison with different studies of Maeder et al., [20]; John et al., [ 10] showed that sepsis patients with elevated troponin levels had higher APACHE II scores and higher mortality. One of those studies Mannam et al., [21] who found that mortality was significantly higher in cTnI positive septic patients $(45.4 \%$ vs. 7 . $7 \%, p<0.04)$.

Despite mortality was significantly higher in cTnI negative group but cTnI level was not found to be an independent predictor of mortality and still APACHE II, SOFA scores were found to be predictor of mortality; these results are similar to the study done by Smith et al., [22] for higher mortality in cTnI positive group but didn't prove its nrediction of mortalitv
Finally, as regard relation between outcome and $1{ }^{\text {st }}$ sample cTnI in each group; the study on the hand revealed that there was no statistical significant difference between outcome and 1 st sample cTnI in Group with positive cTnI, Group II with non-elevated cardiac troponin I.

Moghadam and Shibli [23] also demonstrated that elevated cTnI levels measured upon admission were not associated with increased morbidity or mortality rates. Cardiac troponin does not independently predict mortality beyond that provided by APACHE II.

Sepsis patients with high cTnI levels are usually more critically ill while had the same chance to adverse outcome and less mortality and cTnI level is not a predicator of mortality, further studies in larger patient populations must establish whether elevated troponin may be used as an independent mortality risk factor for intensive care patients without ACS.

\section{Conclusion:}

Based on our results we recommend for further studies in larger patients and longer period of follow-up to emphasize our conclusion. Sepsis patients with high cTnI levels are usually more critically ill while had the same chance to adverse outcome and less mortality and cTnI level is not a predicator of mortality, further studies in larger patient populations must establish whether elevated troponin may be used as an independent mortality risk factor for intensive care patients without ACS.

\section{References}

1- SINGER M., DEUTSCHMAN C.S., SEYMOUR C.W., SHANKAR HARI M., ANNANE D., BAUER M., et al.: The Third International Consensus Definitions for Sepsis and Septic Shock (Sepsis-3). JAMA, 315 (8): 801, 2016.

2- ANGUS D.C. and VAN DER POLL T.: Severe sepsis and septic shock. The New England Journal of Medicine, 369 ( 9): 840-51, 2013.

3- KAUKONEN K.M., BAILEY M., PILCHER D., COOPER D. J. and BELLOMO R.: Systemic Inflammatory Response Syndrome Criteria in Defining Severe Sepsis. N. Engl. J. Med., 372: 1629-38, 2015.

4- SOONG J. and SONI N.: Sepsis: Recognition and treatment. Clinical Medicine. 12 (3): 276-80. Archived from the original on 23 September 2015, 2012.

5- SANDS K.E., BATES D.W., LANKEN P.N., et al.: Academic Medical Center Consortium Sepsis Project Working Group. Epidemiology of sepsis syndrome in 8 academic medical centers. JAMA, 278: 234-40, 2007.

6- FROMM R.E.: Cardiac troponins in the intensive care unit: Common causes of increased levels and interpreta- 
7- MEHTA N.J., KHAN I.A., GUPTA V., JANI K., GOWDA R. M. and SMITH P.R.: Cardiac troponin I predicts myocardial dysfunction and adverse outcome in septic shock. Int. J. Cardiol., 95: 13-7, 2004.

8- MININO A.M., HERON M.P., MURPHY S.L., et al.: Disease Control and Prevention on National Centre For Health Statistics National Vital Statistics System. Deaths: Final data for 2004. Natl. Vital. Stat. Rep., 55: 1-119, 2007.

9- NAPOLITANO L.M.: Sepsis 2018: Definitions and Guideline Changes. Surgical Infections, 19 (2): 117-25. doi: 10.1089/sur.2017.278, 2018.

10- JOHN J., WOODWARD D.B., WANG Y., YAN S.B., FISHER D., KINASEWITZ G.T. and HEISELMAN D.: Troponin-I as a prognosticator of mortality in severe sepsis patients. J. Crit. Care, 25 (2): 270-5, 2010.

11- LIM W., QUSHMAQ I., DEVEREAUX P.J., et al.: Elevated cardiac troponin measurements in critically ill patients. Arch. Intern. Med., 166: 2446-54, 2006.

12- SALAH ELDEEN S., KHALAF M.M. and EL HADIDY K. E.: Cardiac Troponin I as a Marker of Sepsis Severity and Mortality Prediction. Med. J. Cairo Univ., 80 (2): 167-72. www.medicaljournalofcairouniversity.com Cardiac Troponin I as a Marker of Sepsis Severity and Mortality Prediction, 2012.

13- KHALAF M., SALAH ELDEEN S. and ELHADIDY K.: Cardiac troponin I as a marker of sepsis severity and mortality prediction. The Medical Journal of Cairo University, 79: 167-72, 2019.

14- KNAUS W.A., SUN X., NYSTROM P.O., WAGNER D. P., et al.: Evaluation of definitions for sepsis. Chest, 101 (6): 1656-62, 1992.
15- EIDELMAN L., PUTTERMAN D., PUTTERMAN C., SPRUNG C.L., et al.: The Spectrum of Septic Encephalopathy. JAMA, 275 (6): 470-3, 1996.

16- OSTERMANN M., AYIS S., TUDDENHAM E. and LO J., LEI K.: Cardiac Troponin Release is Associated with Biomarkers of Inflammation and Ventricular Dilatation During Critical Illness, Shock, 47 (6): 702-8, 2017.

17- ALTMANN D.R., KORTE W., MAEDER M.T., FEHR T., HAAGER P., RICKLI H., KLEGER G.R., RODRIGUEZ R. and AMMANN P.: Elevated cardiac troponin I in sepsis and septic shock: No evidence for thrombus associated myocardial necrosis. PLoS One, 5: e9017, 2010.

18- GUPTA V., KARNIK N.D. and AGRAWAL D.: SOFA Score and Critically Ill Elderly Patients, Journal of The Association of Physicians of India, 65 (7): 47-50, 2017.

19- AZIZ F., SHARMA M., PENUPOLU S., KHANAL R., DODDI S., et al.: Prognostic Value of Troponin I \& ProBrian Natriuretic Peptide In Patients With Sever Sepsis: A Closed Unit Experience Am. J. Respir. Crit. Care Med.,183: A4681, 2011.

20- MAEDER M., FEHR T., RICKLI H., et al.: Sepsisassociated myocardial dysfunction: Diagnostic and prognostic impact of cardiac troponins and natriuretic peptides. Chest, 129: 1349-66, 2006.

21- MANNAM P., DEVARAKONDA V.S., WITTBRODT E. T., et al.: Association of troponin concentrations with outcomes in sepsis. Chest: 865S: 126, 2004.

22- SMITH A., JOHN M., TROUT R., DAVIS E. and MONINGI S.: Elevated cardiac troponins in sepsis what do they signify? W.V. Med. J., 105 (4): 29-32, 2009.

23- MOGHADAM S. and SHIBLI M.H.: Cardiac TroponinI as A Marker of Outcome in Patients with Sepsis. Chest, 35: 584-8, 2008. 


\section{تقييي تروبونين القلب (آى) كدلالة على الإنتان

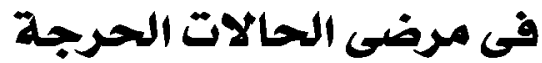

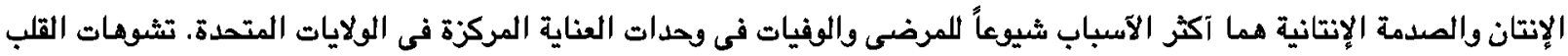

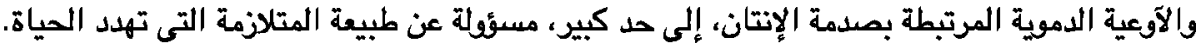

وقد ثبت آن تروبونين القلب هو مؤشر على إصابة عضلة القلب وهو عامل تتبؤ مقبول لإحتشاء عضلة القلب.

على الرغم من آن ترويونين القلب خاص بالقلب، يبدو آن إطلاقه لا يقتصر على الآحداث المتعلقة بالقلب، والكن يمكن إكتشافه آيضاً فى الآى

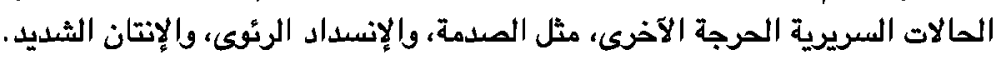

وقد ثبت آن إرتفاع الترويونين القلى فى المرضى الذين يعانفن من الإنتان الثديد والإنتات الثديد والصدمة الإنتانية يشير إلى سوء

التشخيص.

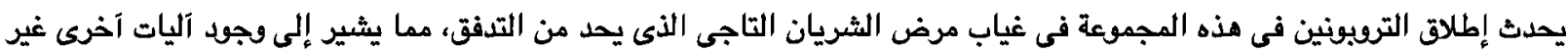

إنسداد الثريان التاجى الخثارى، وربما فقدان عابر فى سلامة الغشاء مع إطلاق تروبونين لاحق آو إصابة خثرة وعائية.

الميزة الرئيسية ل ترويونين القلب هى قدرتها على إكتشاف تلف خلايا عضلة القلب التى لا يمكن إكتثافها من خلال طرق الإنئ الإنيم التقليدية،

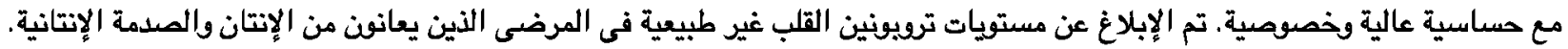

تهدف الدراسة إلى دراسة القيمة التتبؤية لتروبونين القلب على الوفيات والمضاعفات السلبية فى المرضى الذين يعانون من الإنتان والمدمة

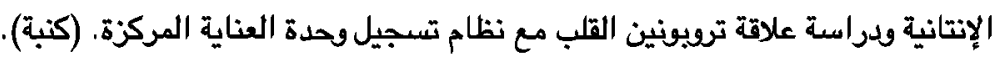

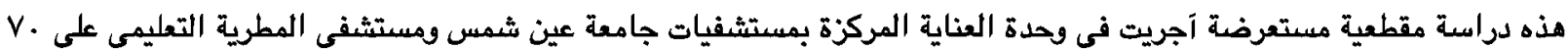

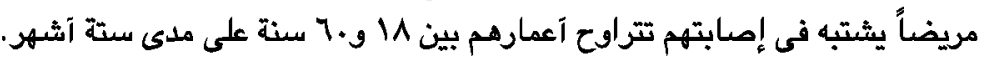

